\title{
Bieolmaging
}

\section{Making Movies on a Weekend}

\author{
Steve Paddock and David McDougal \\ University of Wisconsin, Madison, \\ Madison, WI, USA
}

Digital movies have become popular for displaying micrographs at seminars (14) or on the Web (6). It is now practical to produce acceptable presentations using digital images collected with various microscopes and affordable laboratorybased microcomputers. This column will describe some of the methods that are now available to the microscopist for producing digital movies and the requirements for their display using different media including video, digital slide presentations and the Web.

\section{Image Sources}

The images can be from any digital source, or they can be translated into a digital image. For example, one of our recent forays into the world of digital movie making contains single digital images of fixed and fluorescently labeled butterfly wing imaginal discs collected with a confocal laser scanning microscope (1), a movie sequence of living butterflies collected on location in Costa Rica with a digital video camera and single photomicrographs digitized with a slide scanner (Figure 1).
There are basically two different types of image files produced by microscopes that are suitable for inclusion in a digital movie. These are single image files, such as an image of a fixed and fluorescently labeled embryo that has no motion associated with it, or movie files, such as a time-lapse sequence of a living embryo that has plenty of motion associated with embryonic cell movements.

Single image files can be displayed as a series of static im ages in a digital slide presentation or processed further into an animation or 3-D representation. Regardless of the source, all files should be converted to the final resolution of the digital video, for example, $640 \times 480$ pixels. The tagged image file format (TIFF) is relatively stable for digital imaging files, and it is compatible with most animation software. To avoid artifacts, it is recommended that the source images be no smaller than the resolution of the video, and in the TIFF format.

The most convenient method of standardizing single im age files is to export them from the source programs used for their acquisition into an image manipulation program such as PhotoShop $^{\circledR}$ (Adobe Systems, Mountain View, CA, USA). Indeed the acquisition software of many digital cameras is available as a direct Plug In to PhotoShop.

PhotoShop can read many file formats, although the files may have to be passed through a file conversion program such as Graphic Converter ${ }^{\circledR}$ (Lemke Software, Peine, Germany) to convert them into a readable format. In addition, if the dimensions of the images are known, a series of RAW images can
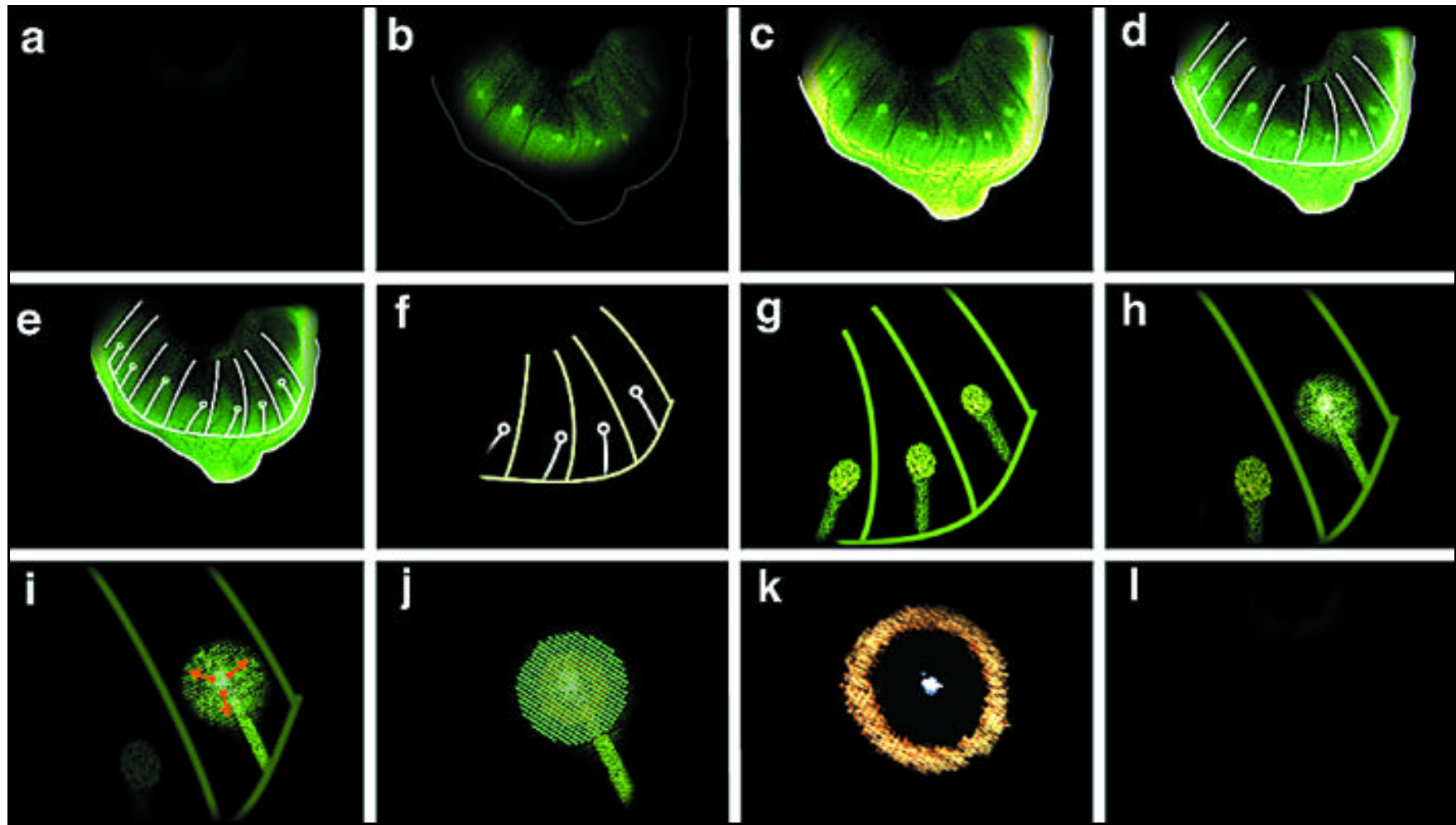

Figure 1. Sample frames extracted from a short digital movie sequence on focal determination of the eyespot during butterfly wing development (1). The sequence begins with a fade from black $(\mathrm{a}-\mathrm{c})$. The subcompartments of the butterfly wing imaginal disc are highlighted with a simple animated line drawing on a different video track that fades to the drawing only ( $\mathrm{d}-\mathrm{f}$ ), and the image is zoomed to a single eyespot field, which is highlighted with red arrows (g-i). Red arrows denote the direction of a signal from the center of the source, which is implicated to have a role in the development of the eyespot of the adult butterfly ( $\mathrm{j}$ and $\mathrm{k}$ ). Finally, the movie fades to black ( $\mathrm{k}$ and $\mathrm{l})$. 


\section{Bi.lmaging}

be imported into PhotoShop (using versions 5.0 and higher) and saved as TIFF files using the "Actions" feature (3). It should be noted that PhotoShop does not open movie files.

Images can be easily resized in PhotoShop, and several can be formed into a single composite image using "Layers". The brightness, color balance and sharpness of the images can also be adjusted (usually using the Unsharp Mask filter). The "Actions" feature is useful for performing identical manipulations on several images when matching colors and backgrounds for the final movie.

There are several different kinds of movie files that are produced from microscopes. These include time-lapse series of living specimens, such as a developing embryo or a Z-series of a fixed and stained embryo from multiple-photon, confocal or wide-field deconvolution microscopes. The Z-series files are further processed into 3-D representations using third-party 3-D reconstruction algorithms that produce movie files of the 3-D image viewed from different angles (15).

4-D datasets are Z-Series collected over time from a living specimen (12). These data sets can also be converted into a format that is suitable for display in a movie presentation. Here, programs are available that allow the user to follow a labeled structure through the series of $\mathrm{Z}$ planes over time and to export a subset of the total dataset as a sequence of selected images as a QuickTime ${ }^{\circledR}$ (Apple Computer, Cupertino, CA, USA) movie file (12). Alternatively, each time point can be processed into a 3 -D representation or stereo pair, and each processed image of the series is subsequently placed into the movie (8).

\section{Adding Motion}

Single image files can be animated using 2-D and 3-D graphics programs. 2-D graphics can be produced using a program such as Flash ${ }^{\circledR}$ (Macromedia, San Francisco, CA, USA), which creates vector-based images that can be changed along a timeline. In addition, this program is valuable for drawing and animating diagrams. Morphing software such as Elastic Reality ${ }^{\circledR}$ (Avid Technology, Tewksbury, MA, USA) has been used for modeling developmental events $(4,9)$. For example, images of Drosophila wing imaginal discs that were fixed and stained at different stages of development and imaged using confocal microscopy were morphed into a continuous developmental sequence (Figure 2).

Programs such as Premiere ${ }^{\circledR}$ (Adobe Systems), After Effects ${ }^{\circledR}$ (Adobe Systems) or Final Cut Pro ${ }^{\circledR}$ (Apple Computer) can be used for animating both still images and movies. Here, the still frames from PhotoShop can be scrolled, rotated, overlapped or animated in many ways using filters nested in the programs themselves or as Plug Ins from third-party vendors. Using these programs, multiple images can be placed on different tracks, given different "motion paths" and animated over a specified number of frames (Figure 1). These short sequences are usually previewed at low resolution (e.g., $240 \times$ 180 pixels).

3-D graphics and animations of still images can be produced using programs such as Maya ${ }^{\circledR}$ (Alias Wavefront, Toronto, Canada). 3-D programs manipulate not only the objects themselves in 3-D but also the lighting and environment surrounding the object (Figure 3).

\section{Editing}

The production of digital movies is computer intensive. Ideally, a Macintosh ${ }^{\circledR}$ G4 or at least a 300-MHz Microsoft ${ }^{\circledR}$ Windows $^{\mathrm{TM}}$ computer with as much hard disk space and RAM as one can afford is recommended or at least the mini-
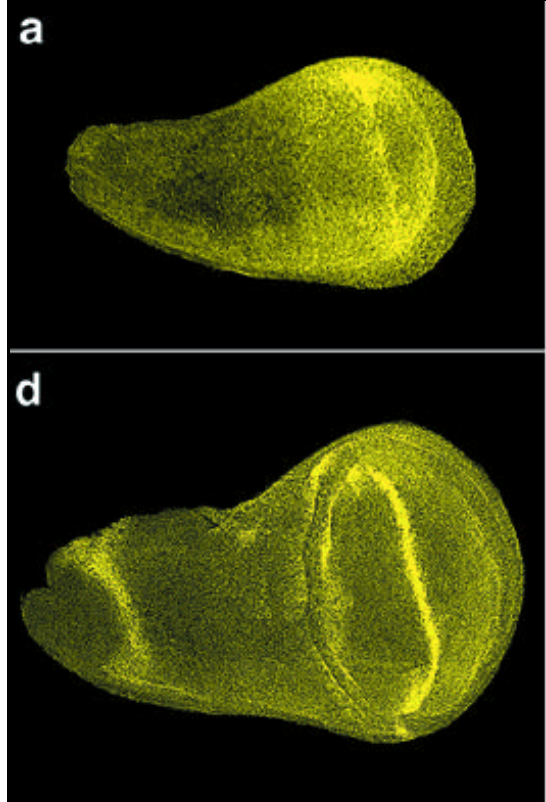
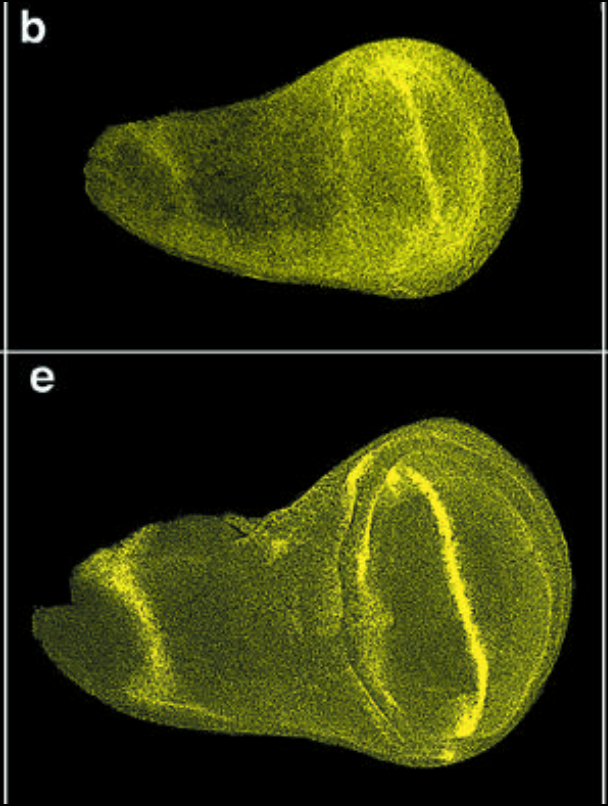
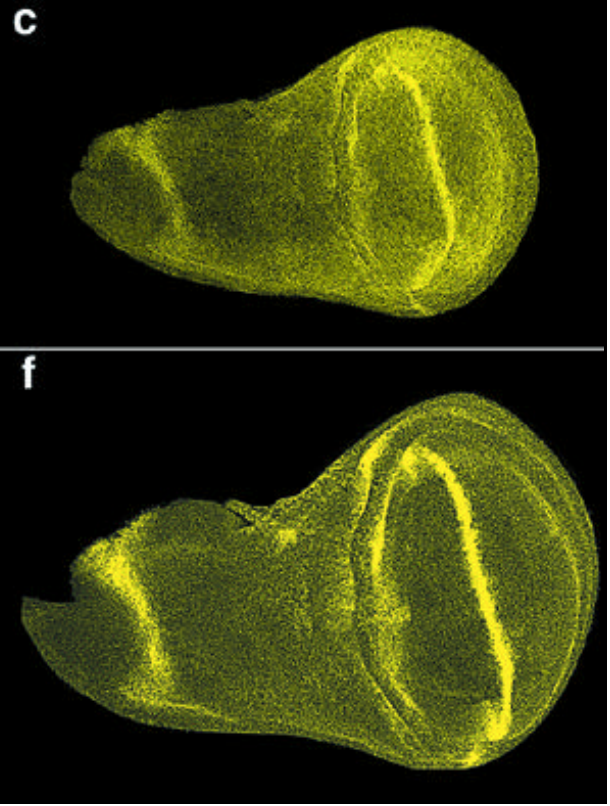

Figure 2. Several frames extracted from a morphed sequence of wingless expression during the development of a Drosophila wing imaginal disc from the second (a) through the third instar (f). Images a and $f$ are actual confocal micrographs, whereas images b-e were intermediaries between a and $f$ generated by the morphing program. 


\section{Bielmaging}

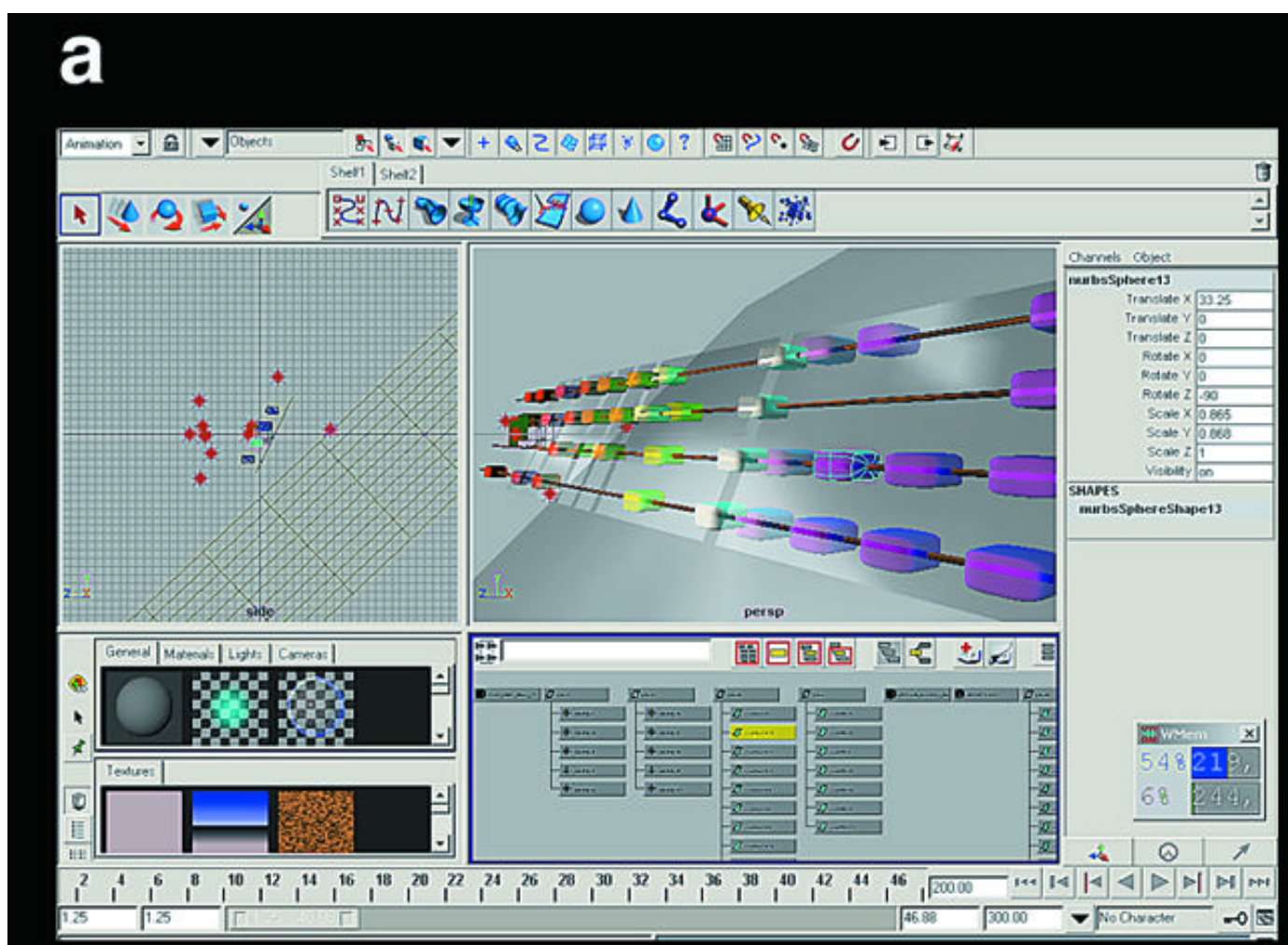

\section{b}

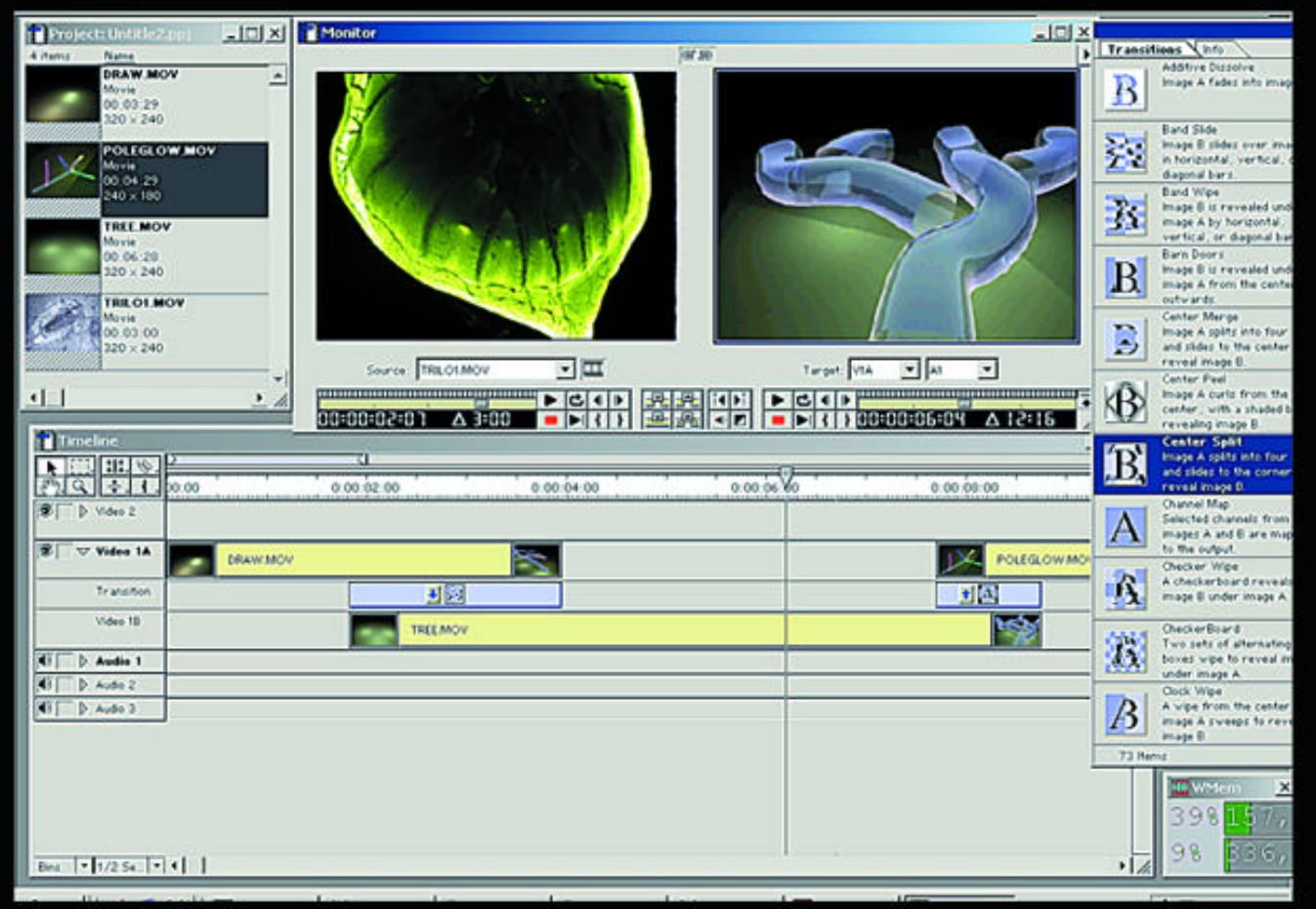

Figure 3. Two examples of software interfaces. (a) Maya produces 3-D graphics of images. In this example, a 3-D graphic of a homeobox gene. (b) Premiere setup for movie making showing the video and audio tracks in use (bottom box). Here, three movie sequences are connected with two fades. The two images are a single image imported from PhotoShop and a single frame from a 3-D movie imported from Maya. 
mum that is specified by the program. The final movies may be edited and compiled in many different nonlinear editing programs (2). Two of the more popular ones that are relatively easy to use, affordable and produce high-quality digital effects for various media are Premiere and Final Cut Pro. These programs will accept both still image files from PhotoShop and most movie file formats.

Still images and movie files are imported into a project folder within the program and placed into a timeline where they can be cut and positioned to the user's liking (Figure 3). A transition can be selected from a list of many (there are currently more than 70 different ones in Premiere) that are used to replace hard cuts between the sequences. Several sequences can also be overlaid (up to 99 movie tracks can be overlaid in the current version of Premiere). A soundtrack can be added, and the volume and placement can be edited so that specific movements and transitions in the movie can be cued to it. As in the video tracks, audio tracks can be filtered and overlaid.

Once the timeline of the movie is complete, all of the transitions and fades between sequences are smooth, the titles have been added and the sound has been edited (usually within Premiere or Final Cut Pro), the movie is processed into the format of its display medium. The hardware requirements for this rendering step depend on the final destination of the movie - to video, the Web, a digital presentation using a laptop, a CD or a DVD.

\section{Rendering to Video}

Rendering to video can be accomplished using Premiere or Final Cut Pro. This is the most computer-intensive step of the entire process because each frame should be rendered at both full frame resolution $(640 \times 480$ pixels $)$ and time resolution (30 frames/s) to avoid pixilated images when the movie is displayed on a TV monitor or projected onto a large screen. As an example, a segment of NTSC video at full frame resolution in 24-bit color at 30 frames/s takes up $27 \mathrm{Mb}$ hard disk space/s video (4). Therefore, it is typical to use a compression/decom pression routine (CODEC) during the rendering step to reduce the file sizes and maintain the quality of the final movie.

It is essential to estimate the final size of the rendered movie so that adequate hard disk space is available to place the movie file as it is being rendered. If space is not available, the computer will crash during the rendering process, which can become both time consuming and frustrating. A large-capacity external hard disk that is dedicated to rendering movie files and can be easily transported between computers is strongly recommended for storing the final movie file as it is being rendered to video. This is a much safer option than rendering the movie file directly onto other external devices such as a JAZ disk.

Many of the latest Macintosh ${ }^{\circledR}$ computers are available with a digital video card or Firewire ${ }^{\circledR}$ (Firewire, Austin, TX, USA) built in. The computer is connected via the Firewire port (IEEE 1394), ideally to either a digital video camera or a digital videotape recorder where the movie is recorded onto digital videotape. A video home system (VHS) or SVHS videotape recorder is then connected to the digital video recorder or digital video camera via RCA or S-Video jacks, and recordings are made onto standard VHS videotape.
Table 1. Web Resources

Programs
www.adobe.com
www.adobe.com/print/features/zdjunsharp/main.html
www.apple.com/quicktime/qtvr/
www.elasticreality.com/
www.macromedia.com/software/flash/
rsb.info.nih.gov/nih-image/
www.webreference.com
Digital Video Hardware
www.dv.com
www.videoguys.com
www.terran.com
www.dvcentral.com
Movies
www.neuro.uoregon.edu/doelab/lineages/
www.clontech.com/whatsnew/specialoffers/gfpinmotion/
gfpCD.html
pantheon.cis.yale.edu/ wfm5/gfp_gateway.html
www.welc.cam.ac.uk/ brandlab/movies/movies.html
sdb.bio.purdue.edu/dbcinema
www.molbio.wisc.edu/carroll/

\section{Movies on the Web}

Digital movies destined for the Web are necessarily of much lower resolution and size than those destined for video because of the Web's current bandwidth restrictions (13). At the present time, a good rule-of-thumb is to restrict the final size of the movie to less than $5 \mathrm{Mb}(10)$. The size and quality of the movie are competing factors for Web-based content, and Media Cleaner Pro ${ }^{\circledR}$ (Terran Interactive, San Jose, CA, USA) is a valuable program that compresses movie files and images to a small size while maintaining relatively good quality.

In general, no additional hardware is required to create a movie for the Web. Web site management tools such as GoLive $^{\circledR}$ (Adobe Systems) or Dreamweaver ${ }^{\circledR}$ (Macromedia) are used to mount the movies onto a Web server. These programs can integrate movie formats such as QuickTime or Realvideo (Internet.com, Darien, CT, USA) with all the features that are needed to link multiple pages together. Many examples of digital video and animations that have been produced from various microscopes are now available for viewing on the Web (Table 1) or on CD (7). Moreover, these programs are useful for routine processing and display of digital microscopy data within the laboratory (5). Many journals include digital movies on their Web pages as additions to their papers (11).

\section{Digital Presentations}

The size of the movie files for a digital presentation depend on the capacity of the computer used to present them, al- 
though they are usually similar in size to those destined for the Web. If short video sequences are included in a presentation using a program such as Microsoft PowerPoint ${ }^{\circledR}$, there are no additional hardware requirements for adding the sequences to the program. PowerPoint currently will not open all movie file formats, so the movie should be saved in .AVI format if PowerPoint for Windows will be used. Most presentations can fit onto a single CD (up to $650 \mathrm{Mb}$.), which makes this an especially convenient method for presenting a seminar "on the road".

For digital presentations, the images are projected onto a large screen in an auditorium using a digital projector that is usually controlled by a laptop computer. The hardware for this is now within the budgets of many laboratories and is available in many seminar rooms and conference facilities. All recently available digital projectors have a "VGA in" that will connect to a "VGA out" on the laptop.

If the host institution has digital projection equipment and software that is compatible with your movie presentation (check well ahead of the seminar), then all you need to take for the seminar is the PowerPoint file of the complete presentation on a $\mathrm{CD}$ or, more safely, the $\mathrm{CD}$ and your laptop. Moreover, text slides can be easily updated, and images and video sequences can be inserted into a PowerPoint presentation at any time-in some cases, just minutes before a seminar.

\section{CD or DVD?}

CDs allow movie files to be viewed faster than if they are downloaded remotely from the Web, especially if a 72X CD drive is available. Alternatively, the files can be downloaded to the hard disk of the computer. CDs are currently the best means of backing up movie files when working with short movies, that is, less than the $650-\mathrm{Mb}$ capacity of a single CD. The ISO 9660 format is convenient for this process because both Macs and PCs can read it.

DVD technology does not currently have the same level of standardization as CD technology. For example, any music CDs can be played in $90 \%$ of all CD players, whereas a DVD made in a DVD-ROM drive will not currently play in any normal DVD player without an expensive DVD authorizing station, although this is likely to change in the future.

\section{Conclusions}

The production and display of digital movies using com mercially available desktop computers with improved and affordable software and projection devices are now practical options for the microscopist. Computer packages that are fully equipped for digital movie making using fast computers with plenty of RAM and gigabytes of hard disk space are available for the import, production and export of digital videos. Images from microscopes and elsewhere can now be combined and displayed in a digital movie production at an acceptable resolution either on video, as a digital presentation or on the Web. Digital movies are important vehicles for the display of visual information collected with microscopes, and the presentations can range in complexity from a simple digital slide show of still images to a fully integrated movie extravaganza.

\section{Acknowledgments}

The movie sequence in Figure 1 was animated by Eric Allin and that of Figure 2 by Eric Buth. The images were kindly provided by Julie Gates and Jim Williams, and thanks to Sean Carroll for the good vibes.

\section{REFERENCES}

1.Brakefield, P.M., J. Gates, D. Keys, F. Kesbeke, P.J. Wijngaarden, A. Monteiro, V. French and S.B. Carroll. 1996. Development, plasticity and evolution of butterfly eyespot patterns. Nature 384:236-242.

2.Capria, F. 1999. Software NLE's grow up. DV October issue, p. 26-44.

3.Halder, G. and S.W. Paddock. 1998. Presentation of confocal images. Methods Mol. Biol. 122:373-384.

4.Hazen, E. 1998. Morphing confocal images and digital movie production. Methods Mol. Biol. 122:421-441.

5.Ladic, L.A. 1998. The use of Internet graphics software for the processing and display of digital microscopy data, p. 189-205. In J.E. Celis (Ed.), Cell Biology: A Laboratory Handbook. Vol. 3. Academic Press, San Diego.

6.Ladic, L.A. and A.M.J. Buchan. 1996. Microscopy animations on the Web. Trends Cell Biol. 6:76-78.

7.Matus, A. 1999. GFP in motion CD-ROM. Trends Cell Biol. 9:43-85.

8.Mohler, W.A. and J.G. White. 1998. Stereo-4-D reconstruction and animation from living fluorescent specimens. BioTechniques 6:1006-1012.

9.Paddock, S.W., P.J. DeVries, E. Buth and S.B. Carroll. 1994. Morphing, a new graphics tool for animating confocal images. BioTechniques 16:448-452.

10.Peck, W. 2000. Twelve ways to speed up your PhotoShop 5.5 Web work. Adobe Mag. 11:46-47.

11.Schmid, A., A. Chiba and D.Q. Doe. 1999. Clonal analysis of Drosophila embryonic neuroblasts: neural cell types, axon projections and muscle targets. Development 126:4653-4689.

12.Thomas, C.F. and J.G. White. 1998. Four-dimensional imaging: the exploration of space and time. Trends Biotechnol. 16:175-182.

13.Waggoner, B. 2000. Getting the best from your Web movies in Premiere. Adobe Mag. 11:41-45.

14.Waterman-Storer, C.M., S.L. Shaw and E.D. Salmon. 1997. Production and presentation of digital movies. Trends Cell Biol. 7:503-506.

15. White, N.S. 1995. Visualization systems for multidimensional CLSM im ages, p. 211-254. In J.B. Pawley (Ed.), The Handbook of Biological Confocal Microscopy, 2nd Ed. Plenum Press, New York.

Address correspondence to Dr. Steve Paddock, Howard Hughes Medical Institute, Dept. Molecular Biology, University of Wisconsin, 1525 Linden Drive, Madison, WI 53706, USA. e-mail: paddock @facstaff.wisc.edu or David McDougal, Dept. Medical Illustration and Photography, University of Wisconsin, Madison.e-mail:djmcdoug@ facstaff.wisc.edu

\section{Suggestions for contributions to the Biolmaging feature are welcomed by its editor, Dr. Steve Paddock (paddock@facstaff.wisc.edu)}

